\begin{tabular}{c}
\hline INTELIGENCIA ARTIFICIAL \\
http://journal.iberamia.org/ \\
\hline
\end{tabular}

\title{
Applying ensemble neural networks to analyze industrial maintenance: Influence of Saharan dust transport on gas turbine axial compressor fouling
}

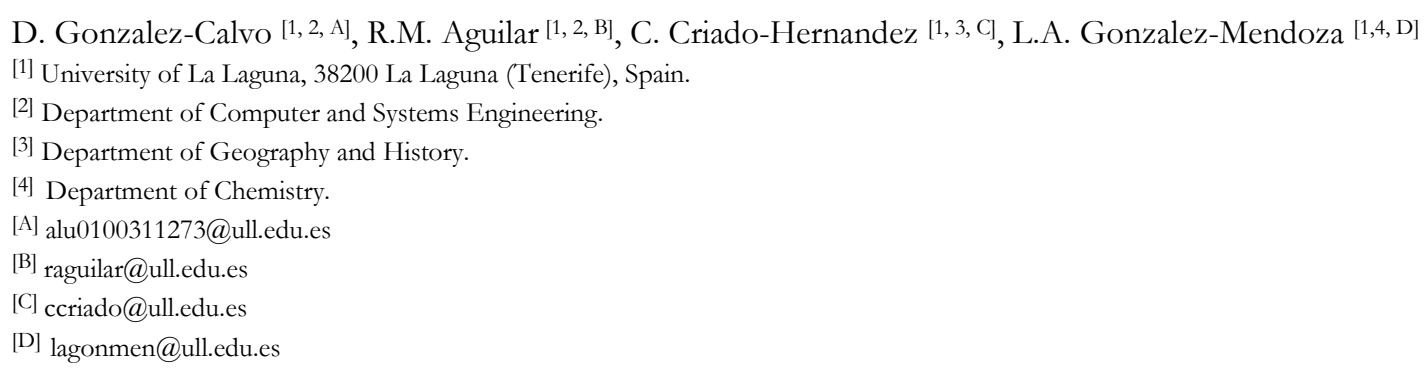

\begin{abstract}
The planning of industrial maintenance associated with the production of electricity is vital, as it yields a current and future snapshot of an industrial component in order to optimize the human, technical and economic resources of the installation. This study focuses on the degradation due to fouling of a gas turbine in the Canary Islands, and analyzes fouling levels over time based on the operating regime and local meteorological variables. In particular, we study the relationship between degradation and the suspended dust that originates in the Sahara Desert. To this end, we use a computational procedure that relies on a set of artificial neural networks to build an ensemble, using a cross-validated committees approach, to yield the compressor efficiency. The use of trained models makes it possible to know in advance how the local fouling of an industrial rotating component will evolve, which is useful for maintenance planning.
\end{abstract}

Keywords: Gas Turbine, Compressor Fouling, Neural Networks Ensemble, Saharan dust, Canary Islands.

\section{Introduction}

A typical gas turbine assembly for generating electricity, as seen in Figure 1, basically consists of a gas generator section and an energy conversion section. The gas generator section consists of a compressor, a combustion chamber and a turbine, which extracts enough energy to drive the compressor. The result of this is a gas at high temperature and pressure at the turbine outlet. Different types of gas turbines can be designed by adding several components at the inlet and outlet to the gas generator. 


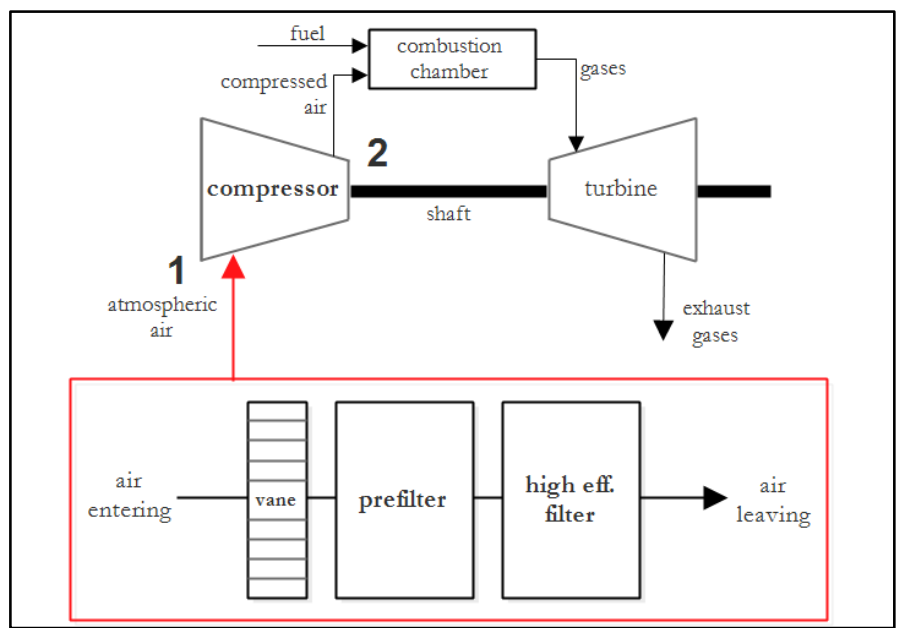

Figure 1. General diagram of a Gas Turbine for electricity generation with a multi-stage inlet air filtration system.

In order for a gas turbine to work properly, large volumes of air have to be compressed. The flow path in an axial flow compressor is parallel to the axis of rotation. The axial flow compressor consumes between $55 \%$ $65 \%$ of the power produced by the turbine section in the gas turbine [1].

To adapt gas turbines to a wide variety of environments and maximize their efficiency and reliability, it is necessary to process the air they intake (Figure 1). Even in relatively clean environments, a gas turbine can ingest a large amount of foreign matter every year whether or not this creates a problem depends on the quantity of this material, its mechanical properties and its chemical composition [2]. Compressor fouling is typically caused by airborne salt, industrial pollution, mineral deposits, or airborne materials [3].

Due to its design and to the large amount of air consumed, any gas turbine is sensitive to air quality. Filtration is applied to provide protection against the effects of contaminated air that can degrade the performance and life of the gas turbine: erosion, scaling, corrosion and obstruction of the cooling duct. Therefore, adequate filtration is vital to this technology [4], [5].

The conventional structure of a filtration block at the compressor inlet of a gas turbine consists of a prefilter stage and a set of high-efficiency filters [4]. Depending on the type of filtration system used, small particles in the $0.1-2.0 \mu \mathrm{m}$ range could enter the turbine [5].

These particles are too small to cause erosion problems, but they are the right size to adhere to the blade surfaces and cause fouling. A study of dirty compressors has revealed contamination on both the suction and pressure sides of compressor blades. Fouling identified in the field has shown that only the first few stages are affected by deposits, and that the absence of centrifugal force in the stator blades leads to more severe fouling in comparison to the gas turbine rotor [2], [6]. The study by Aker revealed that fouling can progress in around 40 to $50 \%$ of the compressor stages [7].

The fouling that occurs gradually and exponentially as the operating hours of the turbine increase, will affect the first stages of the compressor blades, reducing parameters such as the inlet air flow, and its compressor discharge temperature and pressure[8], [9]. This will result in an increase in fuel consumption and, ultimately, in the heat rate of the gas turbine[3]. Losses derived from compressor fouling are classified as recoverable, since they can be eliminated through washing. There is a percentage of losses that, depending on the hours of operation, become "non-recoverable" and can only be fixed with a long outage and/or by replacing components [10], [11]. It is estimated that between $70-85 \%$ of the recoverable losses of a gas turbine are linked to compressor fouling [12]. 
The main solution is to do a series of off-line washes and to replace the inlet filter elements in order to reset the thermal parameters to their initial conditions. Operating hours are proportional to this fouling factor, and reach their critical value at around 2000-2500 hours [13], [14]. According to Tarabrin et al., fouling tends to occur during initial operation and roughly follows an exponential law, stabilizing after 1000 to 2000 hours [6].

Tarabrin also observed the following relationship due to compressor fouling. A drop in the discharge pressure of around $5.5 \%$ resulted in a loss of electricity generated at the gas turbine output of $13 \%$, and a increase in heat rate of $6 \%$.

In the study carried out by Inamul et al., the drops in compressor efficiency obtained were on the order of $0.8 \%$ and $0.5 \%$ over the hours of operation (1290h) [15].

In another case, the fouling that diminishes the intake air flow by $5 \%$ will cause a drop in compressor efficiency of about $2.5 \%$. This will result in an 8 to $10 \%$ drop in the power output and a $4 \%$ drop in thermal efficiency [16].

Other researchers have detailed and modeled the problem of compressor fouling, such as [17]-[19], where, for example, Mohammadi et al. simulated changes in compressor fouling depending on different electricity production scenarios under both full and partial loading, reaching the conclusion that the fouling was not the same, and thus changed with each gas turbine production scenario [20].

To evaluate the above, some studies have applied artificial neural network models to the prognosis and diagnosis of gas turbines. Kiakojoori et al. proposed using dynamic neural networks to measure the degradation due to compressor fouling; however, they stated that their solution could be improved by employing ensemble learning strategies with neural networks [21]. Tahan et al. developed a multi-net artitial neural network (ANN) model where they achieved the best performance in an architecture with a single hidden layer with training with Bayesian regularization, before eventually combining the different partitions generated by the cross-validation method with $k$-fold=4. They applied this model to detect compressor fouling and their research showed that the employment of several networks in the form of a multi-nets model instead of using a single network model promotes diagnostic reliability and ensures the detection of faults at an earlier stage [22].

The main concept behind an ensemble learning model is the simple intuitive idea of a committee of experts working together to solve a problem. All members contribute their own experience and initiatives, and the group as a whole can choose to uphold or reject a new idea on its own merits [23]. Other similar works[24][26], have studied the application of ensemble methods to gas turbines.

In line with Saviozzi et al., the ensemble-averaging method is usually implemented to achieve more accurate results than a single ANN [27]. The main idea of this method is to train different networks and combining their outputs in order to have a better prediction. In terms of the methodologies used in the ensemble averaging approach, Siddhartha et al. observed that the predictive algorithms outperformed the generalized additive model [28]. Weng et al. shows in their experiment with ensemble methods, an improvement of on average $30 \%$ in test performance metrics compared to a single two-layer neural network with the same characteristics and features, including number of neurons [29]. Other recent studies conducted that use a combination of multiple artificial neural networks through an ensemble method have exhibited better performance and yielded improved results. This can be useful to deal with real world applications [30]-[33]. Amozegar et al. propose working with dynamic ensembles to monitor gas turbines; specifically, they distinguish between a type of homogeneous ensemble that uses the same learning algorithm, and a heterogeneous one in which each member of the ensemble uses a different learning algorithm. In both cases, the performance exceeds that of the models that were trained individually [17]. 
Accordingly, the main contributions of this paper are summarized below:

(1) We will develop an artificial neural network (ANN) model where we apply a cross-validated committee (CVC) ensemble architecture so that we can estimate compressor fouling, taking into account the condition of the intake filters and the composition of an axial compressor in a gas turbine for power generation in the Canary Islands.

(2) In order to evaluate the strength of our adaptation of ensemble learning via cross-validation, we propose comparing different learning models for regression problems and compare different $k$-fold combinations to observe its evolution in statistical performance indices.

(3) We will measure the effect that calima, which is the local term used in the Canary Islands to refer to dust from the Sahara Desert, has on the thermal efficiency of the compressor and its inlet filters. For this purpose we will use the final ANN-CVC, where we propose a series of simulation matrices with the result of compressor cleaning activities in any season of the year depending on hours of operation, local climatic conditions and the current operating conditions of the machine, which will allow us to study how the degradation due to fouling evolves over time.

The paper is organized as follows. Section 2 describes the calculation methodology for both the isentropic efficiency of the compressor and for constructing the Sahara sand totalizer, how the hours of operation between maintenance activities are calculated, the data structure for training and testing, the main architecture of the ANN, its training algorithm and the computational architecture designed. Section 3 presents our results and validates the processing of the original data and proposes the maintenance simulation matrices. The paper concludes with a final assessment of the objectives achieved.

\section{Methods}

The general computation procedure in this study is represented by Figure 2 where, the calculated isentropic efficiency of the compressor is estimated using actual humidity, atmospheric pressure, turbine inlet temperature, gross electric output, operating hours without cleaning the compressor, differential pressure at the inlet filters and a totalizer for sand of Saharan origin, which was designed from an on-site analysis of a sample of dust from a gas turbine compressor. All the data were collected in the south of the island of Tenerife. 


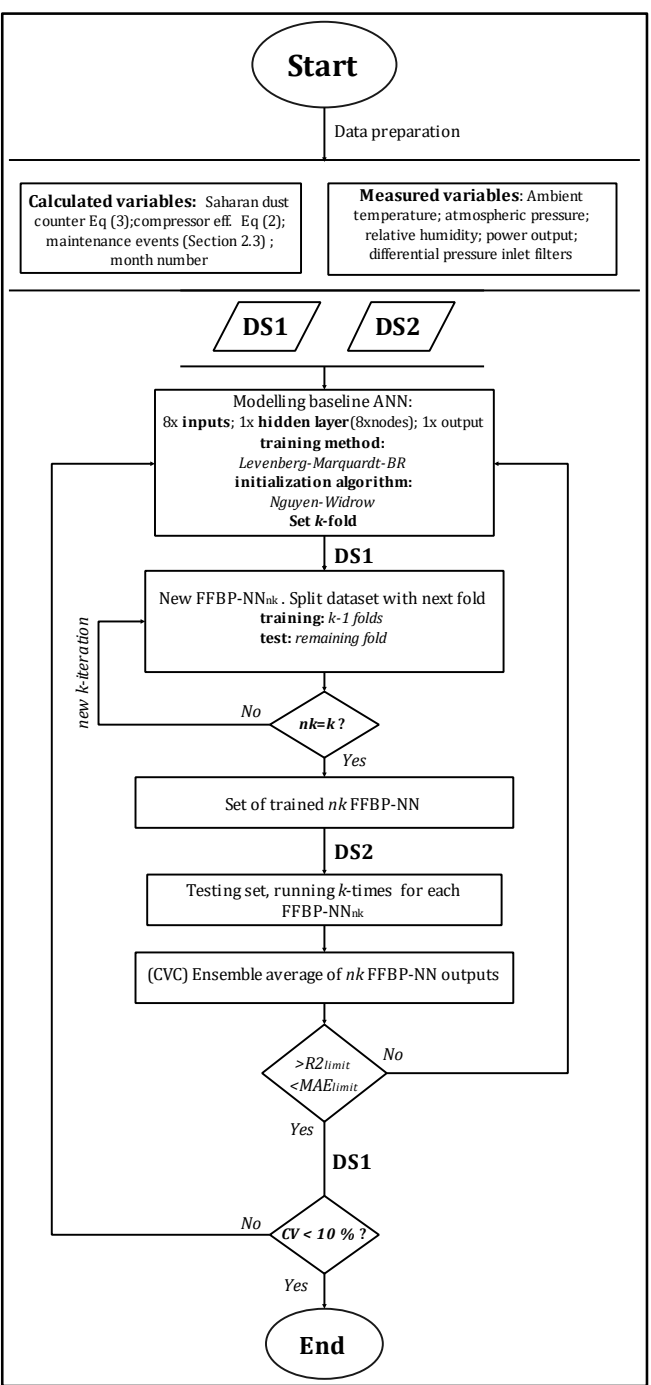

Figure 2. Flowchart of the main procedure used in this work. Section 2.1 to Section 2.4.

\subsection{Study design}

The data set is divided into two main blocks (Figure 3). The first consists of an initial dataset (DS1) containing the input and output data from January 2013 to December 2017, with one sample per minute for the environmental input data. This set will be used exclusively to conduct the entire training process.

The second set of independent data (DS2), not used to train the neural network ensemble, is from the January 2018 to December 2018 timeframe and also contains input and output data. It is intended exclusively for the testing and results verification phase.
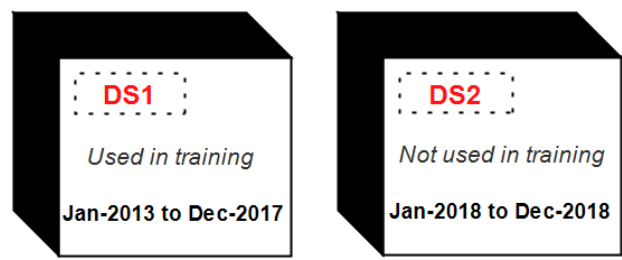

Figure 3. Data sets DS1 and DS2, used to train and test the neural network. 


\subsection{Data collection}

The efficiency of a machine, whether it produces or consumes work, is expressed as a relationship between the work that would be transferred in the ideal transformation and that transferred in the actual transformation. Since the operation of turbo machines is practically adiabatic, the process that is taken as ideal is the isentropic process and the resulting efficiency is called isentropic efficiency. To have a measurement close to the thermal reality of a gas turbine, a method of calculating the isentropic efficiency of the compressor is proposed (Eq. (2)), where the adiabatic coefficient to be calculated takes into account the specific heat, represented by the fraction of humidity in the environment and the range of actual average temperatures measured in the compressor (Eq. (1))[34], [35].

$$
\gamma=\frac{\left(c p_{a}\left(\frac{w}{m_{a}}\right) \cdot c p_{h}\right)}{\left(c v_{a}\left(\frac{w}{m_{a}}\right) \cdot c v_{h}\right)}
$$

Where $w$ is the absolute moisture flow and $m_{a}$ the mass flow of dry air entering the compressor. To approximate the real value of the incoming moist air, we define a set of specific heats $(c p): c p_{a}$ at a constant pressure of dry air, $c p_{h}$ at a constant pressure of the fraction of moist air, $c v_{a}$ at a constant volume of dry air and $c v_{h}$ at a constant volume of moist air. All of them are obtained from the real average of compressor inlet and outlet temperatures [36].

Grouping them yields the calculated isentropic efficiency of the compressor:

$$
\boldsymbol{\mu}_{\boldsymbol{c o}}=\frac{\left(\left(T_{1} \cdot\left(\frac{P_{2}}{P_{1}}\right)^{\frac{\gamma-1}{\gamma}}\right)-T_{1}\right)}{\left(T_{2}-T_{1}\right)} \cdot 100
$$

For this process, the actual measurements of $P_{2}($ bara $)$ and $P_{1}($ bara $)$, which are the compressor inlet and discharge pressures respectively, are available. The inlet pressure takes into account the pressure drop due to fouling of the filter bank. In addition, $T_{1}$ and $T_{2}$ are the temperatures at the compressor inlet and outlet, respectively. The value of $\gamma$ is determined in Eq. (1).

The concentration of Saharan dust $(\mu \mathrm{gr} / \mathrm{m} 3)$ on the surface of the island of Tenerife was obtained from the BSC-DREAM8b v2.0 model (Figure 4a).

To complete the historical dataset, the data from the BSC model was combined with the data obtained by the artificial neural ensemble model for Saharan Dust in the Canary Islands developed by Gonzalez [30].
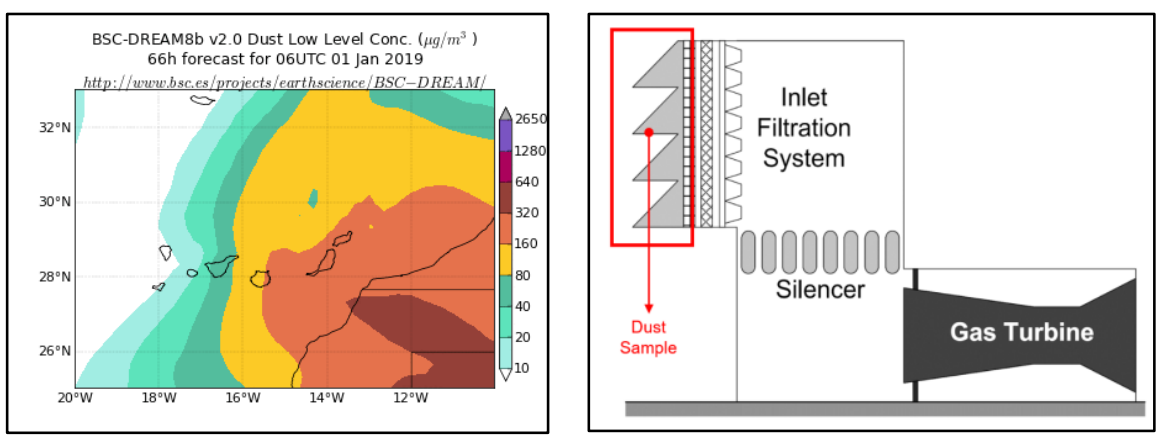

Figure 4a. Data from the BSC-DREAM8b-Dust model for the Canary Islands, operated by the Barcelona Supercomputing Center.

(bttp://wmw.bsc.es/ESS/bsc-dust-daily-forecast). Figure 4b. Sample taken from filter area at gas turbine inlet. Source: adaptation of Guideline for Gas Turbine Inlet Air Filtration Systems [37]. 
A dust sample was taken from the gas turbine, specifically from the inlet filters (Figure $4 \mathrm{~b}$ ), and analyzed. The analysis was done in an external laboratory (CSIC-ICTJ $A$ ), where a diffraction spectrum was obtained using a Bruker D8-A25 diffractometer in a $\theta / \theta$ geometry, equipped with a $\mathrm{Cu} X$-ray tube (wavelength $\lambda=$ $1.5405 \AA$ ), Soller slits and an ultra-fast PSD detector (Lynxeye). A scan from $4^{\circ}$ to $60^{\circ}$ of $2 \theta$ was performed in $0.05^{\circ}$ increments and an equivalent measurement time per increment of 576s. The following working conditions were applied to the tube for the measurements: voltage of $40 \mathrm{kV}$, current of $40 \mathrm{~mA}$.

The diffraction spectra were interpreted using the Bruker Diffrac.SuiteTM program, while the crystalline phases were identified using the PDF-2 database from the International Centre for Diffraction Data (ICDD).

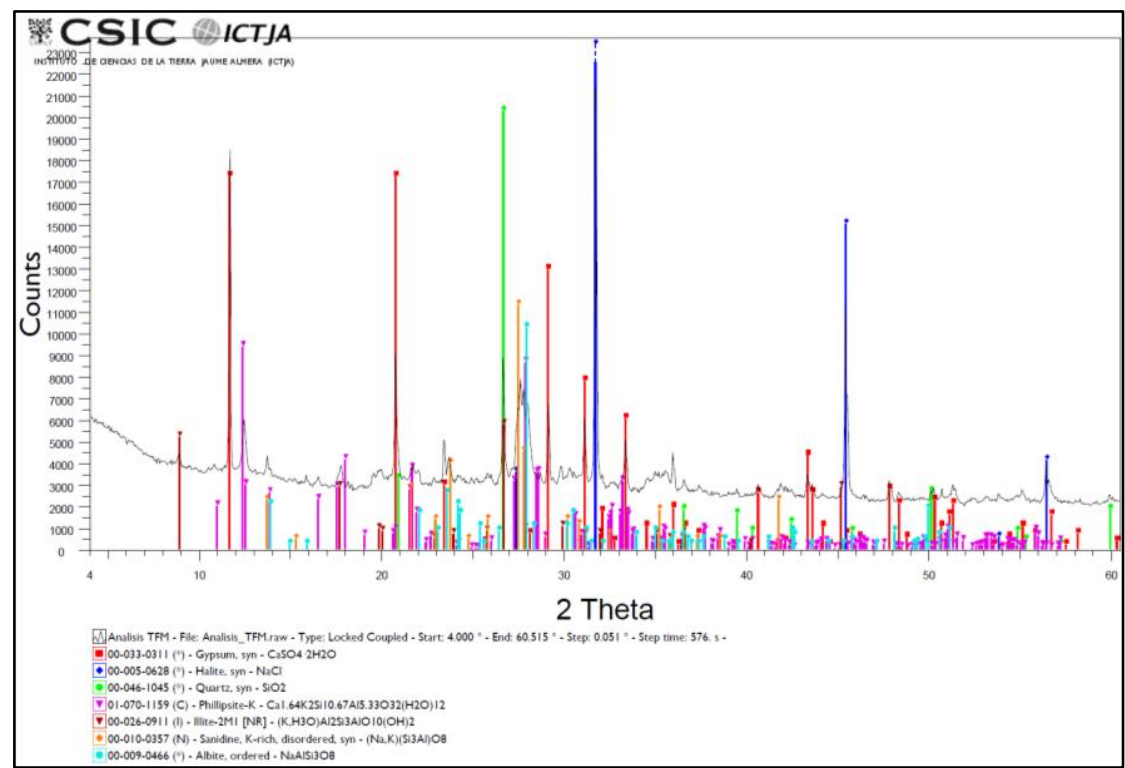

Figure 5. Adaptation of the diffractogram analysis of the sand, prepared by an external laboratory (CSIC-ICTJA), and the resulting composition of the local sample in the filter zone.

The diffractogram of the sample analyzed (Figure 5), reveals the presence of the following crystalline phases: plaster $(15 \%)$, halite $(20 \%)$, quartz $(5 \%)$, feldspar (sanidine $(20 \%)$ and albite $(5 \%))$, the clay phase Muscovite/illite (5\%) and the zeolite phillipsite (30\%).

The local foci were associated with the Macaronesian conditions, and the temporary composition attributable to a one-time human contribution is discarded.

Saharan dust comprises $10 \%$ of the fouling sample taken from the filter bank of the gas turbine. Quartz and Muscovite are common minerals in Saharan sand [38]-[41].

In order to establish a system for tracking Saharan dust that can be used to monitor the accumulation of sand and differentiate all material that does not come from calima, the following accumulation methodology is proposed $t$ with a one-minute frequency:

$$
\boldsymbol{Q}_{\text {dust }}(t)=Q_{\text {dust }}(t-1)+\boldsymbol{a} \cdot\left(\left(\frac{q_{\text {air }} \cdot c_{\text {dust }}}{\rho_{\text {air }}}\right) \cdot 10^{-6}\right)
$$

Where $Q_{\text {dust }}(g r)$ represents the amount of sand from the calima, which accumulates in the gas turbine compressor, $q_{a i r}\left(\frac{k g}{s}\right)$ is the actual total mass of air entering the compressor, $C_{d u s t}\left(\frac{\mu g r}{m 3}\right)$ is the 
concentration of sand measured on the surface, determined in combination with the BSC model for the Canary Islands (Figure 4a), $\rho_{\text {air }}\left(\frac{\mathrm{kg}}{\mathrm{m} 3}\right)$ is the density of the moist air as a function of temperature, relative humidity and atmospheric pressure, calculated using the CIPM-81/91 procedure [42].

Finally, the constant $\boldsymbol{a}(\%)$ represents how much of the sample analyzed in the local area corresponds to Saharan dust. That is, the result of the analysis performed, which yielded a value of $10 \%$.

Maintenance condition is calculated based on the length of time the gas turbine has been running with a gross output power generation above one megawatt electric (MWe) during the entire period studied. An aggregate runtime counter is used that will be reset after maintenance. This means that it will be reset to zero when the compressor is taken off-line and cleaned, after which the counter will begin to accumulate hours of operation until the next cleaning cycle.

The sand counter, and thus the $Q_{d u s t}$ total Eq. (3), will also be reset to zero when the compressor is cleaned.

The actual maintenance tasks associated with changing filters and cleaning the gas turbine compressor adhered to the following historical sequence outlined in Table 1, and will be associated with DS1 and DS2.

Table 1. Record of maintenance done $(\bullet)$ on the gas turbine

\begin{tabular}{l|cccccc}
\hline \multicolumn{1}{c}{ Data set distribution } & \multicolumn{7}{c}{ DS1 } & DS2 \\
\hline Type of Maintenance & 2013 & 2014 & 2015 & 2016 & 2017 & 2018 \\
\hline Offline Washing of Compressor & $\bullet$ & $\bullet$ & $\bullet$ & $\bullet$ & $\bullet$ \\
\hline Replacement of Prefilters & $\bullet$ & $\bullet$ & $\bullet$ & $\bullet$ & $\bullet$ \\
\hline $\begin{array}{l}\text { Replacement of High-Efficiency } \\
\text { Filters }\end{array}$ & $\bullet$ & $\bullet$ & & $\bullet$ \\
\hline
\end{tabular}

\subsection{Training, validation and test procedure}

The type of neural network presented in this paper is defined as a Feedforward Back-propagation Neural Network (FFBP-NN). Starting below, we propose the main architecture of the baseline model. Its basic architecture consists of eight inputs (Ambient temperature; atmospheric pressure; relative humidity; month number; power output; differential pressure inlet filters; operating hours without compressor washing; Saharan dust total counter), a single hidden layer with eight nodes with an activation function that uses the hyperbolic tangent, and an output layer with a linear activation function (Compressor isentropic efficiency) (Figure 6). The number of nodes in the hidden layer was adjusted by adapting the method proposed by Huang, which relates the number of inputs to the output variables using artificial neural models in a hidden layer [43].

For the same DS1 set, and to train it, the data will be divided into the training and testing sub-sets using $k$ fold cross-validation. This division will follow the computational procedure specified in the application of the ensemble method, discussed in this section.

In the data preparation, rows containing constant values were removed and min-max normalization scaling was applied. A technique which provides linear transformation on original range of data [44]. 


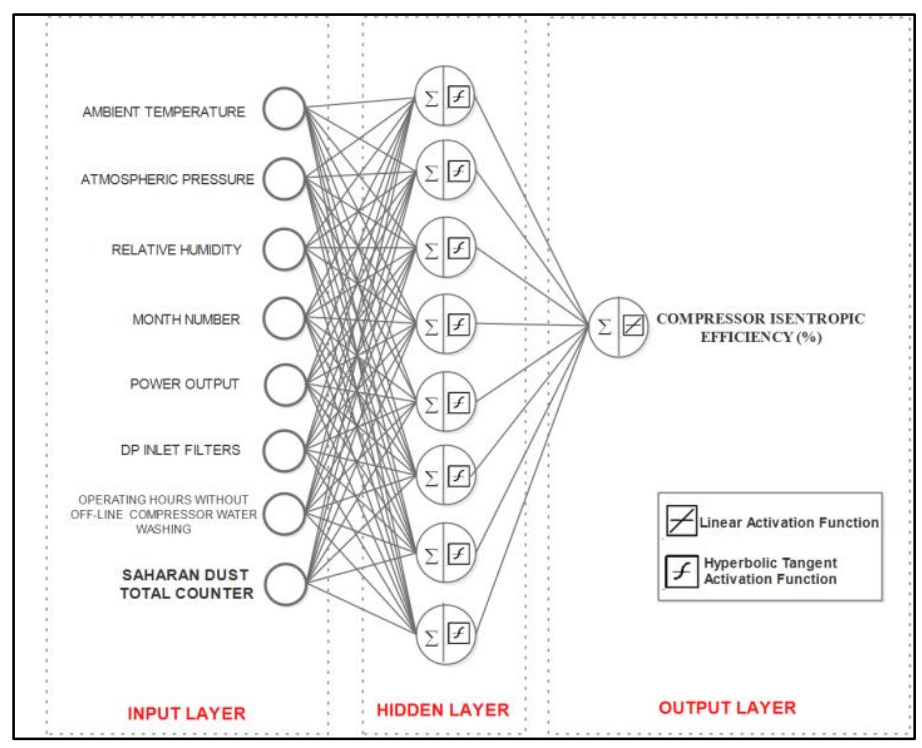

Figure 6. Topology of the main structure of the neural network designed with eight inputs, eight nodes in the hidden layer with a hyperbolic tangent activation function, and one node in the output layer with a linear activation function.

The main optimization method which was applied during the training phase for the entire set of neural networks in this paper is the Levenberg-Marquardt algorithm, modified with Bayesian regularization, was applied as a back-propagation (BP) supervised learning rule and will be used as the basis for the computational procedure proposed [45].

The Nguyen-Widrow algorithm was used to initialize the adaptive weights in each neural network and shorten the training phase [46].

The statistical performance indices used were the determination coefficient $\left(\mathrm{R}^{2}\right)$ and the mean absolute error (MAE). According to Willmott et al., the MAE offers a natural measure of the mean error (unlike RMSE) [47].

The MAE is calculated as follows:

$$
M A E=\frac{\sum_{i=1}^{n}\left|y_{i}-x_{i}\right|}{n}
$$

Where $n$ is the total number of data sampling points, $y_{i}$ is the prediction and $x_{i}$ is the real value.

The coefficient of determination $\left(\mathbf{R}^{2}\right)$ is taken as a measure of the validity of a regression model or a regression estimate. It is calculated as follows:

$$
R^{2}=\frac{\sum_{i=1}^{n}\left(\widehat{y}_{l}-\bar{y}\right)^{2}}{\sum_{i=1}^{n}\left(y_{i}-\bar{y}\right)^{2}}
$$

Where $n$ is the total number of points in the data sample, $\hat{y}_{i}$ is the estimated value of the dependent variable for the $i^{\text {th }}$ observation, as computed with the regression equation, $y_{i}$ is the observed value of the dependent variable for the $i^{\text {th }}$ observation and $\bar{y}$ is the mean of all $n$ observations of the dependent variable.

The closer the regression model's estimated values $\hat{y}_{i}$ lie to the observed values $y_{i}$, the closer the coefficient of determination is to 1 and the more accurate the regression model is [48].

\subsection{Model architecture}

We define a type of ensemble learning with a homogeneous configuration since $n k$ artificial neural network sets will be defined that use the same learning algorithm and feature the same layer architecture proposed in Figure 6 [24]. 
We use the cross-validated committees (CVC) ensemble method with $k$-fold partitioning, similar to that used by Parmanto et al., Xia et al. and Dong et al. [30], [49]-[51].

Specifically, we apply and adapt the computational procedure proposed by Gonzalez et al. (Figure 7), where each fold set will be used to build a FFBP-NN $\mathrm{N}_{\mathrm{nk}}$, where each $n k$ represents the number of the corresponding ANN set associated with the CVC (Figure 7) [30].
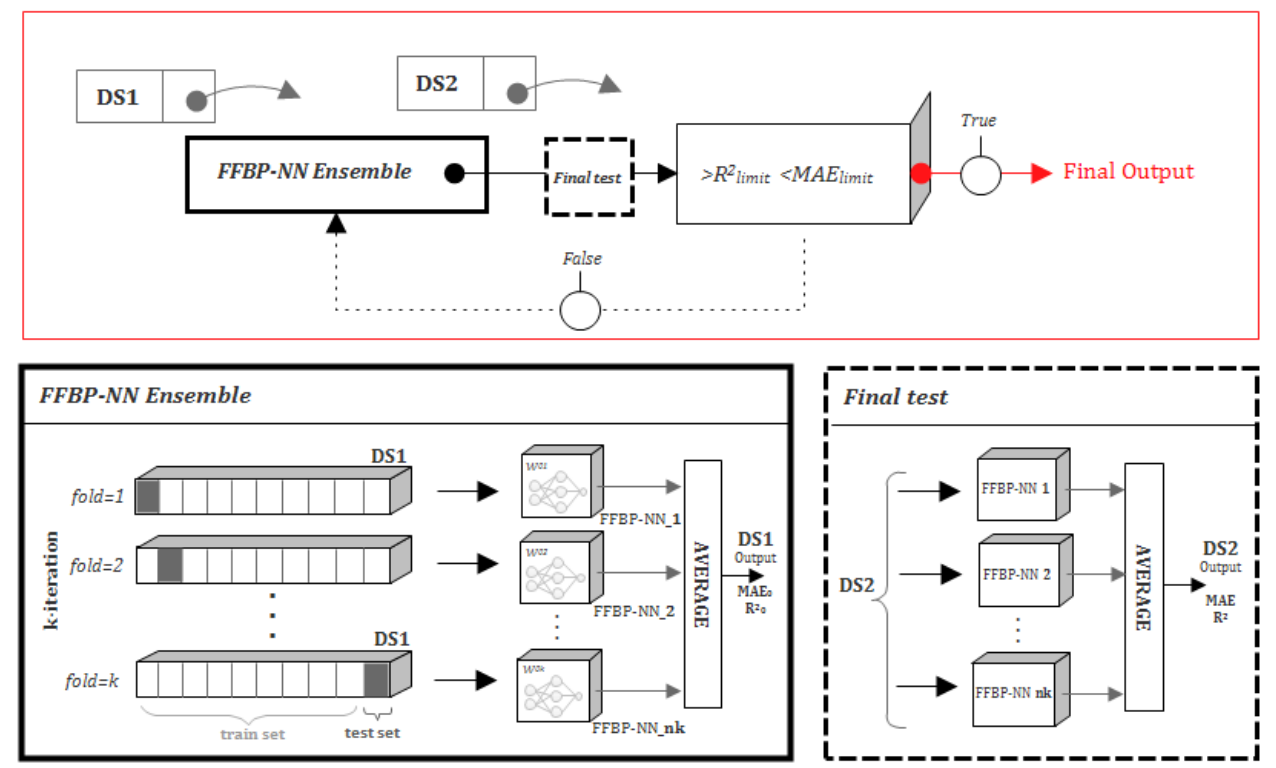

Figure 7. Main diagram of the training algorithm used, with detailed views of the Ensemble Learning k-fold partitioning and Final Test blocks [30].

In order to make this set with different partitions, the $k$-fold cross-validation method is applied to DS1. This method will use k-1 folds to select the training part and the last fold to evaluate it. This algorithm will repeat the process $k$ times, always maintaining a different fold each time for the test phase in each iteration (Figure 2).

This iterative cycle involving DS1 is used to generate each individual set, which is grouped in the corresponding FFBP-NN $\mathrm{nk}_{\mathrm{nk}}$, thus ensuring that all the data contained in DS1 at some point participate in the training and test stages in the CVC.

In our case, each FFBP-NN $\mathrm{Nk}_{\mathrm{nk}}$ is trained using the learning procedure specified in Section 2.3. Moreover, each of the $n k$ outputs obtained will be used to determine the final average of the $n k$ sets, and thus construct the final value that will be evaluated using the statistical performance methods proposed: the mean absolute error $\left(\mathrm{MAE}_{0}\right)$ and the determination coefficient $\left(\mathrm{R}_{0}^{2}\right)$, which result from comparing this estimated average output with the actual output of DS1.

The final test block will be evaluated using the final average value, which results from running DS2 $k$ times for each FFBP-NN $\mathrm{N}_{\mathrm{nk}}$ trained in the previous step with DS1, as long as the safety limits determined by the $\mathrm{MAE}$ and $\mathrm{R}^{2}$ are not exceeded.

It applies the Shewart c-chart for the limit value of the MAE, a statistical quality control method called the control chart for nonconformities, and a limit value of $\mathrm{R}^{2}$ that exhibits a strong degree of correlation, as indicated by Taylor in his research on this correlation coefficient. (Eq. (6)), [30], [52], [53]. Where $\bar{c}$ represents the average value of the DS1 set for the target output variable (Eq. (7)).

$$
\begin{array}{r}
R_{\text {limit }}^{2} \geq \mathbf{0 . 7} \\
M A E_{\text {limit }}=\overline{\boldsymbol{c}}+\mathbf{3} \sqrt{\overline{\boldsymbol{c}}}
\end{array}
$$


The standard division we propose initially for the CVC is $k=10$, which seems optimal in terms of statistical performance for application to this type of ensemble structure. According to Kohavi, the best method to use for model selection is a ten-fold stratified cross validation, even if computational power allows using more folds [54], [55].

This means that for this particular case where $k=10$, each set $n k$ will use $90 \%$ of the set for training and the remaining $10 \%$ for the test phase.

However, we will evaluate the robustness of the CVC by comparing its results, along with the application of other classical regression methods (Table 2). For our comparative case, the Gradient Boosting(GB) and Random Forest $(\mathrm{RF})$ models have been trained for a total of 520 and 300 estimators respectively, following the procedure indicated in Table 2.

The GB and RF methods are tree-based models, used to deal with nonlinear problems. Among various machine learning methods, the GB method proposed by Friedman has been considered as a powerful algorithm with many successful applications for both classification and regression problems in different fields [56].

$\mathrm{RF}$ is a popular machine learning procedure which can be used to develop prediction models. Introduced by Breiman, random forests are a collection of classification and regression trees which are simple models using binary splits on predictor variables to determine outcome predictions [57]. Moreover, in order to define and verify the definitive $k$-fold number in the $\mathrm{CVC}$, we will propose different combinations of the $k$ value, which will be evaluated in Section 3.2.

Table 2. Summary of the comparison study with other computation methods for regression problems

\begin{tabular}{l} 
The compressor performance is calculated using GB, RF, FFBP-NN and three different combinations of the $k-$ \\
fold value through FFBPNN-CVC. \\
\hline For each comparison method, the same data set is used, DS1, $90 \%$ of which is used for training and $10 \%$ for testing. \\
\hline The hyperparameters, numbers of leaves and estimators for the GB and RF models are configured iteratively with \\
until the best configuration is obtained in terms of the statistical performance provided by MAE and R ${ }^{2}$. \\
Finally, all the models are evaluated with DS2, which was not involved in the training phase, to calculate their MAE \\
and $\mathrm{R}^{2}$. The FFBPNN-CVC models are evaluated with DS2, as explained in Section 2.6
\end{tabular}

\subsection{Maintenance simulations}

On the basis of the ensemble models, three types of simulations were defined to observe the results that are obtained for the target output variable (the isentropic efficiency of the compressor) under different environmental and operating scenarios for the gas turbine (Table 3).

Table 3. Summary of the compressor simulations (CS)

\begin{tabular}{cll}
$\begin{array}{l}\text { Maintenance } \\
\text { Simulation }\end{array}$ & \multicolumn{1}{c}{ Main scenarios } & Manipulated variable \\
\hline CS1 & $\begin{array}{l}\text { Six scenarios increasing the operating hours and using the monthly } \\
\text { average value (Dec-18) for the environmental variables. }\end{array}$ & $\begin{array}{l}\text { The degree of fouling }\left(Q_{d u s t} \text { and the }\right. \\
\text { differential pressure of the filters are } \\
\text { increased. }\end{array}$ \\
\hline CS2 & $\begin{array}{l}\text { Six scenarios with different filter differential pressures and using the } \\
\text { monthly average value (Dec-18) for the environmental variables and } \\
\text { 720 operating hours. }\end{array}$ & The degree of fouling $\left(Q_{d u s t}\right)$ is increased. \\
\hline CS3 & $\begin{array}{l}\text { Four scenarios for different output power. The rest of the } \\
\text { environmental variables are set to the average of DS2. }\end{array}$ & $\begin{array}{l}\text { The cleaning counters are reset to zero to } \\
\text { simulate a compressor cleaning. } \\
\text { The average ambient temperature is } \\
\text { modified according to Tenerife season. }\end{array}$ \\
\hline
\end{tabular}


In the first simulation (CS1), the baseline input data is that associated with a snapshot of the gas turbine in the last month of DS2 (December-2018), selecting the monthly average for the ambient temperature, relative humidity, atmospheric pressure and the operating regime of the gas turbine in that period, in terms of gross electric output. On that basis, the total hours of operation will be simulated assuming no compressor cleaning maintenance, as the values of the total Saharan sand counter and the differential pressure values at the filter inlet, in intervals of $+20 \mu \mathrm{gr} / \mathrm{m} 3$ and $+5 \mathrm{mmH} 2 \mathrm{O}$ respectively, are increased for each runtime scenario.

A second simulation was run (CS2) where the total operating hours without washing the compressor was set to a constant value of 720 hours, increasing only the total accumulated sand value for various differential pressure scenarios at the inlet filters.

The third simulation (CS3) shows the result of compressor cleaning depending on the time of year and under different gross electric output conditions in the gas turbine. Four scenarios were chosen where only the temperature of the air at the compressor inlet is adapted to the average conditions of each season. The remaining environmental input variables will be the average of the DS2 set and the sand counter, operating hours and differential pressure of the filters will be reset to their initial values, thus simulating a compressor wash and replaced inlet filters.

\section{Results}

\subsection{Performance analysis for the FFBPNN-CVC ensemble model}

The results predicted by the main ensemble set $(k=10)$ that exceeded the statistical limits proposed in the computational procedure in Section 2.4 are shown (Figure 8). For the training data set DS1, we obtained a regression coefficient $\mathrm{R}^{2} \approx 0.932$ and an absolute mean error $\mathrm{MAE} \approx 0.36 \%$ in terms of the isentropic efficiency of the compressor. The good prediction results of the model were confirmed when the data set DS2, that did not participate in the learning phase yielded an $\mathrm{R}^{2} \approx 0.925$ and an $\mathrm{MAE} \approx 0.53 \%$.

Note that ensemble model not only learned to satisfactorily relate the measurement of the compressor's isentropic efficiency, but when it is informed that at a certain point in the time series, an off-line compressor cleaning event occurred, it reacts with an upward jump similar to the actual figures obtained following cleaning maintenance.

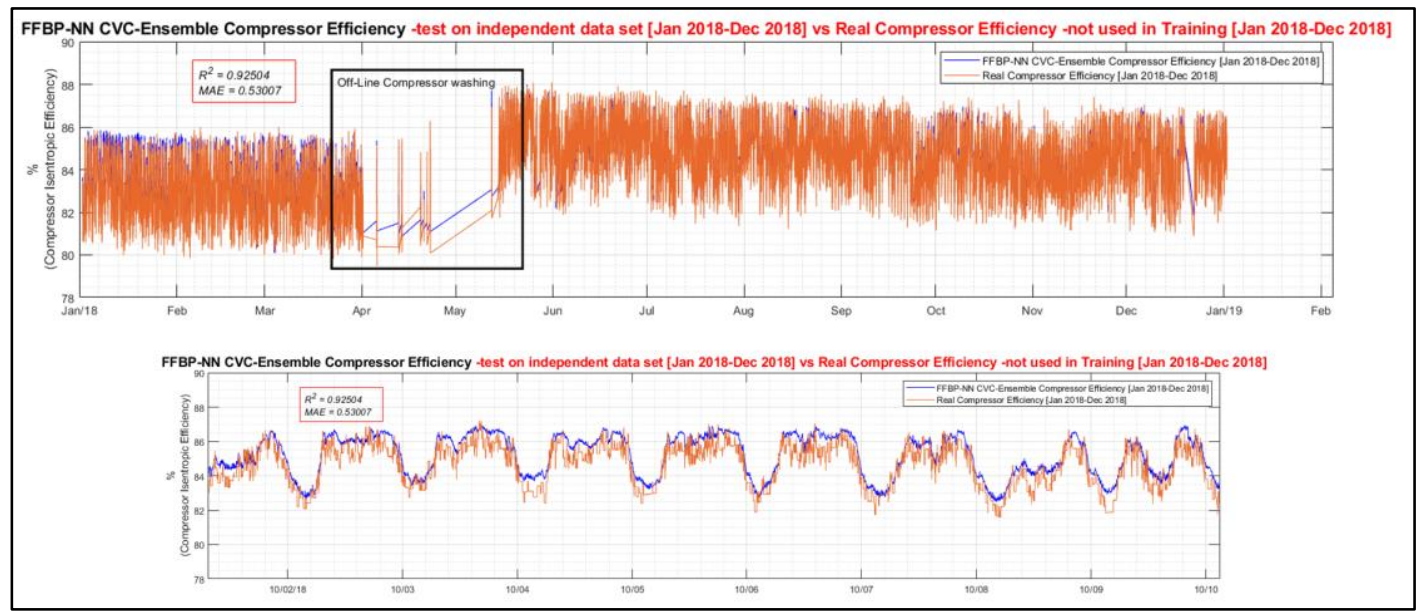

Figure 8. Results and performance of the final FFBPNN-CVC $(k=10)$ model based on real DS2 data. Expanded detail of the learning model for an offline compressor wash. 
Moreover, as indicated in Section 2.3, the output power produced was integrated as an input parameter to the ANN for the baseline architecture, thus reflecting the power oscillations in the final trained model.

It is important to keep in mind that the isentropic performance data for the compressor were calculated for a gas turbine that generates electricity inside an isolated insular electricity grid. Its gross electricity production regime is constrained by the secondary regulation of the electrical frequency on the island [58]. In other words, this gas turbine is not maintained at a constant base load; rather, its output oscillates based on the grid frequency determined by the daily production/demand balance on the island, with a spinning reserve production margin. This is why, when we look at its performance trend (Figures 8) over a reduced time window, we see a constant oscillation in this performance value of approximately $+-4 \%$, resulting from this fact.

\subsection{Evaluation and comparison of regression models}

In comparison with our performance results, Pawełczyk et al. presented a comparison of machine learning predictions models, including a single ANN of three hidden layers for prediction of gas turbine operating characteristics and its potential failures. However, ANN models were reviewed, and their performance checks inferior to Random Forest algorithm-based model [59].

The ensemble average performance, confirms what Amiq et al. observed. That if different models are combined by averaging their output the prediction accuracy is enhanced [60]. However, we note that this reasoning would have to be combined with values of $k$-fold, greater than 10 if we look at the results in terms of MAE and $\mathrm{R}^{2}$ (Table 4).

When comparing statistical returns with other decision tree-based ensemble computation models and a simple FFBP-NN model, we can see how combinations of a CVC ensemble of $k=10$, and $k=15$ can provide the lowest range in statistical performance in relation to the real model (Table 4).

Kohavi and Borra placed their research findings at an optimal value of $k$-fold $=10$. However, for this work and after studying the applied CVC ensemble method, we noted that even with a value of $k=15$, the performance obtained is satisfactory (Table 4) [55], [61].

Table 4. Results obtained for each computation method for regression problems

\begin{tabular}{ccccc}
\hline Computation methods & $\begin{array}{c}\text { DS1 } \\
\text { MAE }\end{array}$ & $\begin{array}{c}\text { DS1 } \\
\mathrm{R}^{2}\end{array}$ & $\begin{array}{c}\text { DS2 } \\
\text { MAE }\end{array}$ & $\begin{array}{c}\text { DS2 } \\
\mathrm{R}^{2}\end{array}$ \\
\hline Gradient Boosting (GB) & 0.34 & 0.89 & $\mathbf{0 . 4 9}$ & $\mathbf{0 . 8 0}$ \\
Random Forest (RF) & 0.38 & 0.86 & $\mathbf{0 . 6 2}$ & $\mathbf{0 . 7}$ \\
FFBP-NN & 0.44 & 0.93 & $\mathbf{0 . 5 4}$ & $\mathbf{0 . 9 1}$ \\
\hline FFBPNN-CVC $(k=5)$ & 0.37 & 0.93 & $\mathbf{0 . 5 1}$ & $\mathbf{0 . 9 0}$ \\
FFBPNN-CVC $(k=10)$ & 0.36 & 0.932 & $\mathbf{0 . 5 3}$ & $\mathbf{0 . 9 2 5}$ \\
FFBPNN-CVC $(k=15)$ & 0.37 & 0.93 & $\mathbf{0 . 5 4}$ & $\mathbf{0 . 9 3}$ \\
\hline
\end{tabular}

\subsection{Simulation based on FFBPNN-CVC to plan maintenance}

The model created is confirmed to have learned the different maintenance tasks involving the off-line cleaning of the compressor and its corresponding dynamics. We were able to carry out various simulations in different operating scenarios and present them in terms of compressor efficiency that yielded a response similar to the real case in order to obtain other relevant results that can help when planning the maintenance of the facility. 


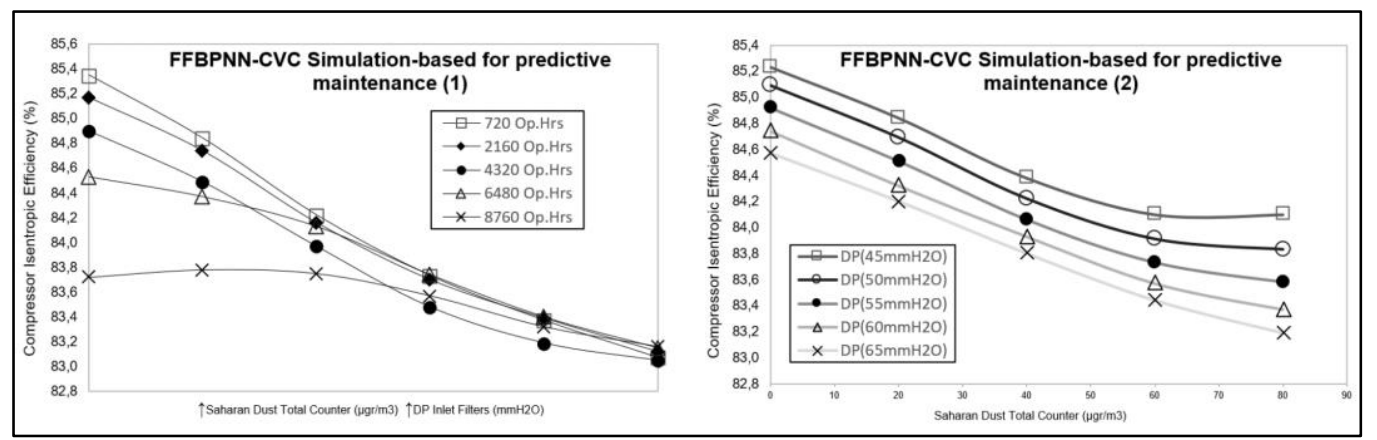

Figure 9. Predictive simulation for planning maintenance (CS1 \& CS2); (1) Operating Hours scenario; (2) DP Inlet Filters scenario.

In the first simulation of CS1, when working with different numbers of operating hours, we see a faster degradation in the compressor as the amount of Saharan sand and differential pressure in the inlet filters increase, for shorter times between compressor washings (Figure 9.1). However, the levels of isentropic efficiency are higher than in those cases where we start with a high number of hours of operation without maintenance. We may thus consider two blocks, the first one between 720 and 4320 hours, where the trend is similar and the efficiency of the compressor loses between 0.4 and $0.5 \%$. The second one comes after 6480 hours of operation without washing, for which the degradation is 1 to $1.5 \%$ below the value that it would have in an operating scenario with the maintenance performed.

In all the scenarios, up to 6480 hours of operation, for values of the sand totalizer with $60 \mathrm{gr}$ and a differential pressure of the filter zone of $55 \mathrm{mmH} 2 \mathrm{O}$, and higher (Table 5), the accumulation of Saharan sand in the compressor and the differential pressure of the inlet filters practically reach a steady state.

Table 5. Results of the simulation matrix for maintenance planning - Operating Hours Scenario

\begin{tabular}{|c|c|c|c|c|c|c|}
\hline \multirow{3}{*}{$\begin{array}{l}\text { S.D. } \\
\text { (gr) }\end{array}$} & \multicolumn{6}{|c|}{ OPERATING HOURS WITHOUT COMPRESSOR OFF-LINE WASHING } \\
\hline & \multirow{3}{*}{$\begin{array}{c}\text { D.P. } \\
(\mathrm{mm} \mathrm{H} 2 \mathrm{O}) \\
40\end{array}$} & 720 & 2160 & 4320 & 6480 & 8760 \\
\hline & & \multicolumn{5}{|c|}{ Compressor Isentropic Efficiency ( $\%)$} \\
\hline 0 & & 85.35 & 85.17 & 84.9 & 84.53 & 83.72 \\
\hline 20 & 45 & 84.84 & 84.74 & 84.49 & 84.37 & 83.78 \\
\hline 40 & 50 & 84.22 & 84.16 & 83.97 & 84.13 & 83.75 \\
\hline 60 & 55 & 83.73 & 83.7 & 83.48 & 83.74 & 83.57 \\
\hline 80 & 60 & 83.37 & 83.39 & 83.19 & 83.4 & 83.32 \\
\hline 100 & 65 & 83.07 & 83.12 & 83.05 & 83.15 & 83.16 \\
\hline
\end{tabular}

In parallel, we noticed that for the same operating scenario after a wash, the efficiency levels of the compressor were around $0.6 \%$ higher when the differential pressure at the inlet filters is decreased by approximately $20 \mathrm{mmH} 2 \mathrm{O}$. This means that the efficiency of the compressor will be greater as long as the filters are adequately maintained.

In this case we also see a stabilization region in the simulation matrix for every level of inlet filter differential pressure calculated (CS2), starting from an approximate sand accumulation of 60 gr (Figure 9.2). From that point, there is a reduction in the compressor degradation rate due to fouling. 
Applying the simulation matrix CS3, proposed in Section 2.5, we find the relationship between the behavior of the compressor efficiency values for each time of year and for each operating scenario of the gas turbine (Figure 10). Note that the winter and autumn seasons exhibit the lowest efficiency following an off-line compressor washing in this period. This result matches that found by Dorta and Criado[38], [39], who identified in these same periods a greater number of calima events in the Canary Islands.

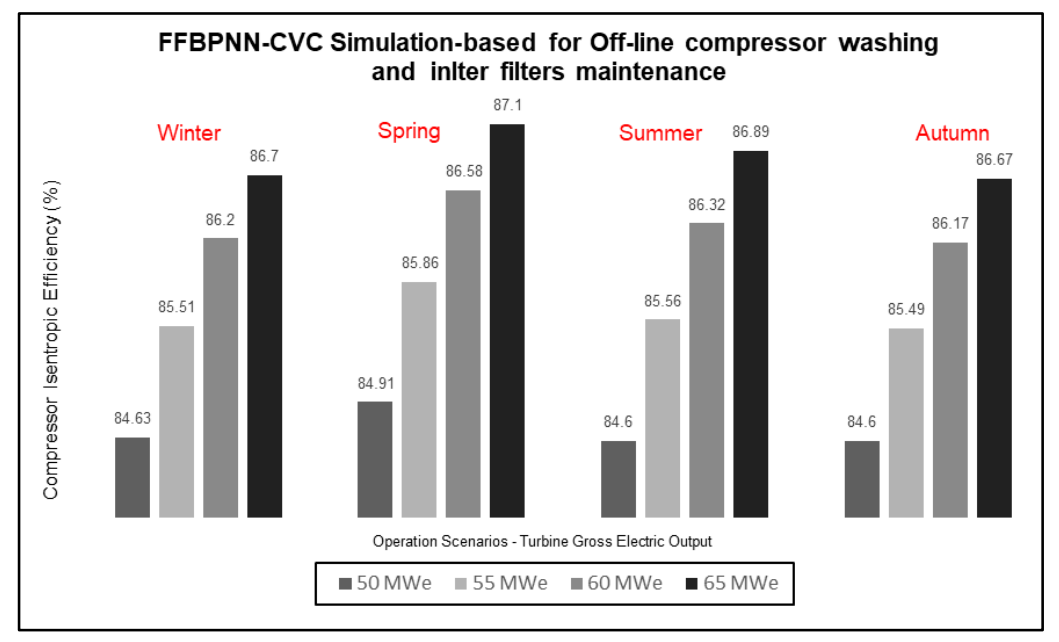

Figure 10. Predictive simulation for maintenance planning (CS3); Scenario for off-line compressor cleaning and filter maintenance by season.

\section{Conclusion}

The models obtained from the FFBBNN-CVC system, after being subjected to new, previously unknown data, exhibited good statistical performance in terms of the isentropic efficiency of the gas turbine compressor.

The studies conducted in this work also showed a direct relationship between the effect that a local weather variable that is typical of the climate of the Canary Islands has on the fouling of a gas turbine compressor. This relationship, defined using a final counter integrated into an artificial neural model with an ensemble architecture, allows for simulations and predictions that reflect the actual evolution of the degradation over time of an industrial rotating component as a function of Saharan dust build-up and operating hours.

Their predictions are thus reflective of the real measurements, even when estimating an actual maintenance activity involving an industrial component, as they are able to predict the evolution of its degradation due to fouling based on the time in service. Moreover, comparing results with other ensemble computation models, our ensemble procedure can provide the more balanced interval in statistical performance in relation to the real model.

Finally, the good results allowed us to simulate different local weather conditions so as to study combinations of maintenance activities and verify their effectiveness at specific times of the year and under different operating scenarios. This provides us with a medium-term snapshot that can be used to optimize and select the ideal frequency for performing new cleaning maintenance activities. 


\section{References}

[1] M. P. Boyce, "Axial-flow compressors," in Gas Turbine Engineering HandbookAnonymous Elsevier, 2006, pp. 275.

[2] E. Syverud, O. Brekke and E. Bakken L., "Axial Compressor Deterioration Caused by Saltwater Ingestion," Journal of Turbomachinery, vol. 129, (1), pp. 119-126, 2007. DOI: 10.1115/1.2219763.

[3] C. B. Meher-Homji and A. Bromley, "Gas Turbine Axial Compressor Fouling And Washing," Texas A\&M University. Turbomacbinery Laboratories, Available: http://hdl.handle.net/1969.1/163249. DOI: 10.21423/R1S66R.

[4] M. Santini et al, "Gas Turbine High Efficiency Filtration Systems," Ge Oil \& Gas, 2013.

[5] R. Kurz and K. Brun, "Fouling Mechanism in Axial Compressors," Journal of Engineering for Gas Turbines and Power, vol. 134, (032401), 2012. DOI: 10.1115/GT2011-45012.

[6] A. P. Tarabrin et al, "ASME 1998 international gas turbine and aeroengine congress and exhibition," in 2014.

[7] G. F. Aker and H. I. H. Saravanamuttoo, "Predicting Gas Turbine Performance Degradation Due to Compressor Fouling Using Computer Simulation Techniques," J. Eng. Gas Turbines Power, vol. 111, (2), pp. 343-350, 1989. DOI: 10.1115/1.3240259.

[8] T. Giampaolo, "Compressor fouling," in The Gas Turbine Handbook: Principles and PracticesAnonymous Fairmont Press, 2003, pp. 205.

[9] A. P. Tarabrin et al, "ASME 1996 international gas turbine and aeroengine congress and exhibition," in 2015.

[10] X. Jiang, T. Lin and E. Mendoza, "Annual conference of the prognostics and health management society," in 2016.

[11] S. Gayraud and R. Singh, "ASME 1997 turbo asia conference," in 1997.

[12] Tech. Rep. GER-3620M (02/15), 2010.

[13] J. -. Stalder, "Gas Turbine Compressor Washing State of the Art: Field Experiences," Engineering for Gas Turbines and Power, vol. 123, (2), pp. 363-370, 2001. DOI: 10.1115/1.1361108.

[14] P. X. Dionysios et al, "Operational optimization of networks of compressors considering conditionbased maintenance," Computers \& Chemical Engineering, vol. 84, pp. 117-131, 2016. DOI:

doi.org/10.1016/j.compchemeng.2015.08.008.

[15] H. Inamul and H. I. H. Saravanamuttoo, "ASME 1991 international gas turbine and aeroengine congress and exposition," in 2015.

[16] I. S. Diakunchak, "Performance Improvement in Industrial Gas Turbines," (93-JPGC-GT-5), 2015. DOI: rg/10.1115/93-JPGC-GT-5. 
[17] H. Hanachi et al, "IEEE international conference on prognostics and health management (ICPHM)," in 03 August 2017.

[18] S. Cruz-Manzo et al, "IEEE international conference on prognostics and health management (ICPHM)," in Dallas, TX, USA, 03 August 2017.

[19] S. Rahimi Malekshan, M. Aliyari Shoorehdeli and M. Yari, " Iranian conference on electrical engineering (ICEE)," in 2-4 May 2017.

[20] E. Mohammadi and M. Montazeri-Gh, "A fuzzy-based gas turbine fault detection and identification system for full and part-load performance deterioration," vol. 46, pp. 82-93, 2015. DOI:

$\mathrm{rg} / 10.1016 /$ j.ast.2015.07.002.

[21] S. Kiakojoori and K. Khorasani, "Dynamic neural networks for gas turbine engine degradation prediction, health monitoring and prognosis," Neural Computing and Applications, vol. 27, 2015. DOI: 10.1007/s00521-015-1990-0.

[22] Tahan Mohammadreza, Z. A. Abdul Karim and Muhammad Masdi, "A multi-nets ANN model for real-time performance-based automatic fault diagnosis of industrial gas turbine engines," Journal of the Brazilian Society of Mechanical Sciences and Engineering, vol. 39, 2017. DOI: 10.1007/s40430-017-0742-8.

[23] B. Baruque and E. Corchado, "Fusion methods for unsupervised learning ensembles," Studies in Computational Intelligence, vol. 322, 2011. DOI: 10.1007/978-3-642-16205-3.

[24] X. Wu and X. Chen, "Internal leakage detection for inlet guide vane system at gas turbine compressor with ensemble empirical mode decomposition," Measurement, vol. 134, pp. 781-787, 2019. DOI: doi.org/10.1016/j.measurement.2018.11.026.

[25] M. Amozegar and K. Khorasani, "An ensemble of dynamic neural network identifiers for fault detection and isolation of gas turbine engines," Neural Networks, vol. 76, pp. 106-121, 2016. DOI: rg/10.1016/j.neunet.2016.01.003.

[26] Z. Zhou and D. Liu, "Prognostics and system health management conference," in 2016.

[27] M. Saviozzi, S. Massucco and F. Silvestro, "Implementation of advanced functionalities for distribution management systems: load forecasting and modeling through artificial neural networks ensembles. electric power systems research," vol. 167, pp. 230-239, 2019. DOI: 10.1016/j.epsr.2018.10.036.

[28] S. Mandal and K. M. Kishore, "Ensemble averaging based assessment of spatiotemporal variations in ambient PM2.5 concentrations over Delhi, India, during 2010-2016," Atmospheric Environment, vol. 224, (117309), 2020. DOI: rg/10.1016/j.atmosenv.2020.117309.

[29] B. Weng et al, "Predicting short-term stock prices using ensemble methods and online data sources," Expert Syst. Appl., vol. 112, pp. 258-273, 2018. DOI: 10.1016/j.eswa.2018.06.016.

[30] D. Gonzalez-Calvo et al, "Multivariate influence through neural networks ensemble: Study of Saharan dust intrusion in the Canary Islands" Applied Soft Computing. DOI: 10.1016/j.asoc.2021.107497.

[31] H. Ashtawy and N. Mahapatra, "Bgn-score and Bsn-score: bagging and boosting based ensemble neural networks scoring functions for accurate binding affinity prediction of protein-ligand complexes," BMC Bioinformatics, vol. 16, (4), 2015. DOI: 10.1186/1471-2105-16-S4-S8. 
[32] N. Alami, M. Meknassi and N. En-nahnahi, "Enhancing unsupervised neural networks-based text summarization with word embedding and ensemble learning," Expert Syst. Appl., vol. 123, pp. 195-211, 2019. DOI: 10.1016/j.eswa.2019.01.037.

[33] Z. Wang, C. Lu and B. Zhou, "Fault diagnosis for rotary machinery with selective ensemble neural networks," Mechanical Systems and Signal Processing, vol. 113, pp. 112-130, 2018. DOI: 10.1016/j.ymssp.2017.03.051.

[34] K. M. Zoran and D. D. Gvozdenac, "Toolbox 6: Thermodynamic and transport properties of moist air," in Applied Industrial Energy and Environmental ManagementAnonymous John Wiley \& Sons, 2008.

[35] M. M. Alhazmy and Y. S. H. Najjarb, "Augmentation of gas turbine performance using air coolers," Applied Thermal Engineering, vol. 24, (2-3), pp. 415-429, 2004. DOI: 10.1016/j.applthermaleng.2003.09.006.

[36] A. P. Santos and C. R. Andrade, "Analysis of Gas Turbine Performance with Inlet Air Cooling Techniques Applied to Brazilian Sites," Aerospace Technology and Management, vol. 4, (3), 2012. DOI: 10.5028/jatm.2012.04032012.

[37] M. Wilcox et al, "Guideline for gas turbine inlet air filtration systems," 2010.

[38] C. Criado et al, "Intercalaciones de polvo sahariano en paleodunas bioclásticas de fuerteventura (Islas Canarias)," Cuaternario Y Geomorfología, vol. 26, (1-2), pp. 73-88, 2012.

[39] P. Dorta et al, "Frecuencia, estacionalidad y tendencias de las advecciones de aire sahariano en Canarias (1976-2003)," Investigaciones Geográficas, (38), 2005.

[40] P. Dorta et al, "Algunas consideraciones sobre la importancia del polvo de origen sahariano en el clima del archipiélago canario y su aporte a las aguas superficiales oceánicas: El episodio de abril de 2002, en el agua y el clima," in III Congreso De La Asociación Española De Climatología, 2002, pp. 13-24.

[41] K. Pye, "Aeolian dust and dust deposits," Academic Press, 1987.

[42] R. S. Davis, "Equation for the Determination of the Density of Moist Air (1981/91)," Metrologia, vol. 29, (1), 1991.

[43] G. Huang, "Learning capability and storage capacity of two-hidden-layer feedforward networks," IEEE Trans. Neural Networks, vol. 14, (2), 2003. DOI: 10.1109/TNN.2003.809401.

[44] S. G. Krishna Patro and K. Kumar Sahu, "Normalization: A Preprocessing Stage," 2015. DOI: 10.17148/IARJSET.2015.2305.

[45] F. Burden and D. Winkler, "Bayesian regularization of neural networks," Literature Review in methods in Molecular Biology, vol. 458, pp. 23-42, 2009. DOI: 10.1007/978-1-60327-101-1_3.

[46] D. Nguyen and B. Widrow, "Neural networks for self-learning control systems," IEEE Control Syst. Mag., vol. 10, (3), pp. 18-23, 1990. DOI: 10.1109/37.55119.

[47] C. J. Willmott and K. Matsuura, "Advantages of the mean absolute error (mae) over the root mean square error (rmse) in assessing average model performance," Climate Research, vol. 30, pp. 79-82, 2005. DOI: $10.3354 / \mathrm{cr} 030079$. 
[48] A. Schneider, G. Hommel and M. Blettner, "Linear regression analysis," Dtsch Aræżtebl Int, vol. 107, (44), pp. 776-782, 2010. DOI: 10.3238/arztebl.2010.0776.

[49] B. Parmanto, P. Munro and H. Doyle, "Improving committee diagnosis with resampling techniques," in Proceedings of the 8th International Conference on Neural Information Processing Systems, 1996, pp. 882-888.

[50] R. Xia, C. Zong and S. Li, "Ensemble of feature sets and classification algorithms for sentiment classification," Inf. Sci., vol. 181, (6), pp. 1138-1152, 2011. DOI: 10.1016/j.ins.2010.11.023.

[51] Y. Dong and K. Han, "A comparison of several ensemble methods for text categorization," in IEEE International Conference on Services Computing, 2004, pp. 419-422.

[52] D. Montgomery, in Introduction to Statistical Quality Control, Sixth edition John Wiley \& Sons Ltd, 2008, pp. 289. ISBN 13: 9780470233979.

[53] R. Taylor, "Interpretation of the correlation coefficient: a basic review," Journal of Diagnostic Medical Sonography, 1990. DOI: $10.1177 / 875647939000600106$.

[54] T. Wong and P. Yeh, "Reliable Accuracy Estimates from k-fold Cross Validation," Transactions on Knowledge and Data Engineering, 201. DOI: 10.1109/TKDE.2019.2912815.

[55] R. Kohavi, "A study of cross validation and bootstrapfor accuracy estimation and model selection," in Proceedings of the 14th International Joint Conference on Artificial Intelligence, 1995, pp. 1137-1143.

[56] J. H. Friedman, "Greedy function approximation: A gradient boosting machine," Annals of Statistics, vol. 29, (5), pp. 1189-1232, 2001.

[57] L. Breiman, "Random Forests," Machine Language, 2001. DOI: rg/10.1023/A:1010933404324.

[58] Yufeng Guo and Daren Yu, "The influence of interconnection of electric power systems on load characteristic and frequency regulation," vol. 70, (1), pp. 23-29, 2004. DOI: rg/10.1016/j.epsr.2003.11.006.

[59] M. Pawełczyk and S. Fulara, "Industrial gas turbine operating parameters monitoring and data-driven prediction," Eksploatacja i Niezawodnosc - Maintenance and Reliability, vol. 22, (3), pp. 391-399, 2020. DOI: 10.17531/ein.2020.3.2.

[60] Atiq ur Rehman and Amine Bermak, "Averaging Neural Network Ensembles Model for Quantification of Volatile Organic Compound," 2019 15th International Wireless Communications \& Mobile Computing Conference (IWCMC). DOI: 10.1109/IWCMC.2019.8766776.

[61] S. Borra and A. Di ciaccio, "Measuring the prediction error. A comparison of cross-validation, bootstrap and covariance penalty methods," Computational Statistics and Data Analysis, vol. 54, (12), pp. 29762989, 2010. DOI: 10.1016/j.csda.2010.03.004. 